\title{
Изготовление $p-n$-переходов в графене и их применение для детектирования терагерцового излучения
}

\author{
(С) Г.Ю. Васильева ${ }^{1}$, Ю.Б. Васильев ${ }^{1 . \uparrow}$, С.Н. Новиков ${ }^{2}$, \\ С.Н. Данилов ${ }^{3}$, С.Д. Ганичев ${ }^{3}$ \\ ${ }^{1}$ Физико-технический институт им. А.Ф. Иоффе Российской академии наук, \\ 194021 Санкт-Петербург, Россия \\ ${ }^{2}$ Aalto University, \\ Fin-02150 Espoo, Finland \\ ${ }^{3}$ Terahertz Center TerZ, University of Regensburg, \\ D-380106 Regensburg, Germany \\ I E-mail: yu.vasilyev@mail.ioffe.ru
}

(Получена 27 декабря 2017 г. Принята к печати 29 декабря 2017 г.)

Рассматривается новый способ формирования латеральных $p-n$-переходов в эпитаксиальном графене с помощью УФ излучения. Применение метода УФ засветки позволяет получить $p-n$-переходы большого размера, которые исследовались в режиме фототока и фотопроводимости при облучении терагерцовым излучением. Обсуждаются механизмы терагерцового фотоответа $p-n$-переходов в графене.

DOI: 10.21883/FTP.2018.08.46225.8809

\section{1. Введение}

В настоящее время исследования уникальных свойств такого перспективного материала, как графен, ведутся по многим направлениям [1]. Наряду с фундаментальными исследованиями изучаются возможности по использованию графена в различных нанотехнологических устройствах [2]. Среди них - оптические детекторы [3], излучатели [4], транзисторы [5], сенсоры со сверхвысокой обнаружительной способностью для химических и биологических применений [6,7] и др. Важной технической задачей является разработка элементной базы электроники на основе графена и, в частности, изготовление графеновых $p-n$-переходов. Имеется несколько способов создания $p-n$-переходов в графене. Рассматриваются варианты использования двойного затвора [8], на который подаются потенциалы разной полярности. Существуют различные методы легирования графена, такие как химические [9], замещения [10-12], плазменные $[13,14]$. Кроме того, было показано, что уровень легирования можно контролировать за счет облучения графена электронным или ионным пучком [15-17]. Также предлагается изготавливать контакты к графену из разных металлов [18], отличающихся по величине работы выхода электронов, чтобы получить асимметричный электрический потенциал в системе металл-графен-металл.

Для создания $p-n$-переходов в эпитаксиальном графене нами использовался новый способ на основе применения УФ засветки графена, что позволяет получить $p-n$-переходы большого размера. В образцах с такими переходами обнаружен фотоответ в режиме фототока и фотопроводимости при облучении терагерцовым излучением.

\section{2. Технология изготовления $p-n$-переходов}

Графеновая пленка была выращена на кремниевой поверхности (Si-грань) подложки 4H-SiC(1000) при отжиге в парах аргона при атмосферном давлении и температуре около $1700^{\circ} \mathrm{C}$ в течение 5 мин. Рост на Si-грани происходит гораздо медленнее, чем на С-грани, и позволяет лучше контролировать толщину пленки, что приводит к формированию однослойного графена на этой грани. С помощью атомно-силового микроскопа была выявлена структура поверхности с периодическими террасами изза небольшой разориентации подложки $\mathrm{SiC}$ от плоскости (0001). Высота этих террас составляла $\sim 0.5$ нм. Толщина пленки оценивалась с помощью оже-спектроскопии, которая подтвердила наличие одного слоя графена. Число слоев можно оценить из соотношения между пиками Si и C, используя метод, описанный в работе [19]. По данным измерений оже-спектроскопии, толщина графена на поверхности карбида кремния в различных образцах может меняться в диапазоне 0.7-1.05 монослоев. Это означает, что процесс роста заканчивается до того, как второй слой графена начинает расти. Имеются данные, что в эпитаксиальной пленке, возможно образование островков двухслойного графена [20], которые не сказываются на свойствах монослойного графена.

Графен, полученный методом термической сублимации на поверхности $\mathrm{SiC}$, имеет ряд особенностей. Одной из них является то, что параметры эпитаксиального графена, как правило, хуже чем в графене, получаемом традиционным методом механического расщепления или „методом скотча“, хотя удается вырастить монослойный графен на С-грани SiC [21] с подвижностями в диапазоне 5000-20000 $\mathrm{cm}^{2} / \mathrm{B} \cdot$ с. Для практического использования, однако, важно, что размеры структур в направлении 
$a$

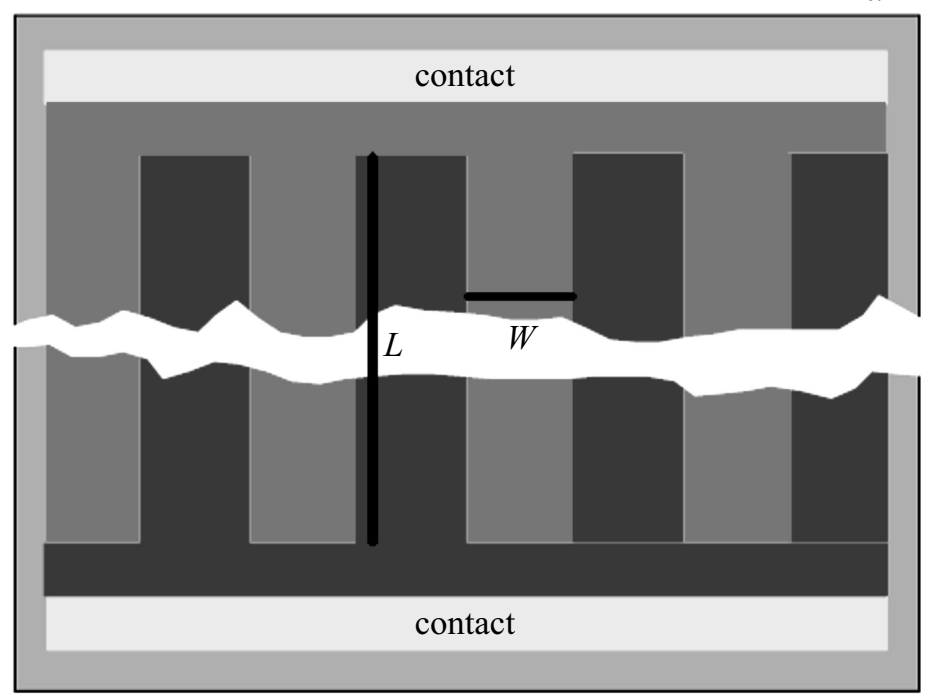

$n^{-} \quad p^{-} \quad$ graphene

SiC substrate $b$

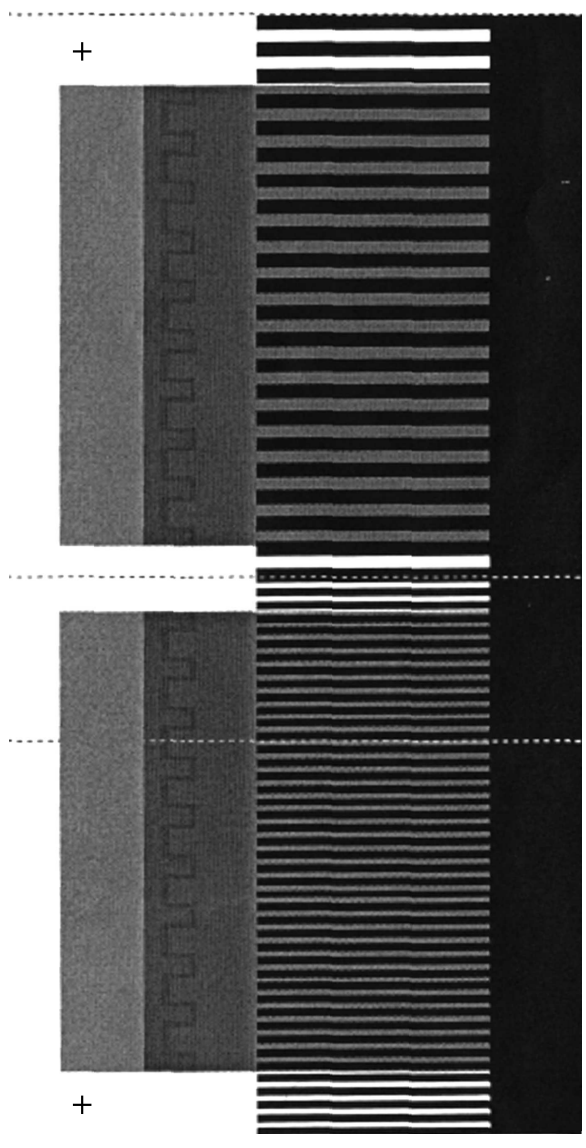

Рис. 1. $a-$ схематическое изображение образца графена с латеральными $p-n$-переходами, возникшими на границе между частями графена с разным типом проводимости. Ширина полосок $W$ равна 100 или 50 мкм, длина $L=2$ мм. $b-$ увеличенная фотография образца графена с латеральными $p-n$-переходами. Темные полоски имеют $p$-тип проводимости, светлые - $n$-тип. Ширина полосок равна 100 мкм для верхнего прибора и 50 мкм для нижнего.

вдоль плоскости графенового слоя достаточно большие и могут составлять несколько сантиметров. Большие размеры пленки графена необходимы, например, для изготовления $p-n$-переходов с увеличенной активной областью, которые могут найти применение в терагерцовой оптоэлектронике. В эпитаксиальном графене, выращенном на поверхности подложки $\mathrm{SiC}$, обычно присутствует двумерный (2D) электронный газ с концентрацией порядка $10^{13} \mathrm{~cm}^{-2}$. Взаимодействие с подложкой $\mathrm{SiC}$ приводит к большой концентрации электронов в слое графена. В качестве источника электронов в графене рассматриваются глубокие доноры, связанные с дефектами в $\mathrm{SiC}$, компенсирующие примеси в $\mathrm{SiC}$ и поверхностные состояния в буферном слое между $\mathrm{SiC}$ и графеном [22].

Перед изготовлением $p$-n-переходов, пластина $\mathrm{SiC}$ с пленкой графена разрезалась на образцы размером $5 \times 5$ мм, которые выдерживались в горячем воздухе, чтобы уменьшить концентрацию носителей заряда в пленке графена [23]. Экспериментально было показано, что молекулы кислорода могут адсорбироваться на поверхности графена, вызывая изменение концентрации носителей заряда. После воздействия кислорода плотность носителей $n$-типа,согласно нашим измерениям, находилась в диапазоне $\left(1.6-3.1 \cdot 10^{11} \mathrm{~cm}^{-2}\right.$, а подвижность оценивалась примерно как (1400-2700) см $2 /$ В с . Затем установленный в держателе образец закрывался двумя слоями полимерных пленок: РММА и ZЕР500, для того чтобы регулировать концентрацию носителей в графене фотохимическим стробированием [24]. С помощью этой методики при облучении глубоким ультрафиолетом через теневую маску поверхности образца были изготовлены $p-n$-структуры, состоящие из графеновых полос, попеременно чередующихся с $p$ - и $n$-типом проводимости. Таким образом, были получены структуры в виде вставленных друг в друга двух гребенок с длинными зубчиками (рис. 1), при этом одна из гребенок облучалась ультрафиолетом для изменения типа проводимости в ней. Доза облучения была выбрана достаточно высокой, чтобы инвертировать тип проводимости в графене из $n$ - в $p$-тип. Ширина зубчиков составляла 100 мкм в одном образце и 50 мкм в другом. 
Вдоль линии соприкосновения зубчиков формируется $p-n$-переход. Тип и степень легирования в гребенках контролировались измерениями эффекта Холла.

Для изготовления надежных и низкоомных контактов использовался двухступенчатый процесс металлизации [25]. Контакты к графену изготавливались методом взрывной литографии. Первый слой металлизации Тi/Au $5 / 50$ нм наносился на поверхность $\mathrm{SiC}$, очищенного от слоя графена с помощью реактивно ионного травления. Второй слой металлизации находился над первой металлизацией и заходил на графен, тем самым образуя омический контакт. Такой способ металлизации был использован для снижения контактного сопротивления. Дополнительно такая методика дает улучшенную адгезию контактных площадок, нанесенных на поверхность карбида кремния (а не на графен), и меньшее повреждение пленки графена, так как соединительные провода крепятся к контактным площадкам первого слоя металлизации, находящимся непосредственно на поверхности $\mathrm{SiC}$.

\section{3. Измерение терагерцового фотоответа в $p-n$-переходе графена}

Для измерения терагерцовой фотопроводимости и фототоков в графене использовался терагерцовый импульсный $\mathrm{NH}_{3}$-лазер $[26,27]$ с накачкой $\mathrm{CO}_{2}$-лазером [28]. Это лазер с перестраиваемой длиной волны. Измерения проводились для длин волн: 90.5 мкм - частота 3.32 ТГц, 148 мкм -2.03 ТГц и 280 мкм -1.07 ТГц. Лазер испускает одиночный импульс продолжительностью 100 нс и пиковой мощностью $\sim 100$ кВт. Длительность импульса и мощность излучения контролировались приемником на эффекте увлечения электронов фотонами $[29,30]$. Измерения с помощью пироэлектрической камеры $[31,32]$ показали, что лазерный пучок является гауссовым с диаметром пятна порядка 1.5 мм.

При освещении $p-n$-перехода терагерцовым излучением длиной волны $\lambda=280$ мкм появляется сигнал фотопроводимости при приложении электрического напряжения к структуре. Вблизи смещения 2В и для положительной, и для отрицательной полярностей смещения обнаружен максимум фотопроводимости (рис. 2), причина появления которого пока не понятна. В целом мы считаем, что сигнал фотопроводимости связан с болометрическим механизмом, который может включать в себя два процесса. Первый из них представляет собой классическое изменение сопротивления образца с температурой под действием лазерного излучения, для которого характерны большие времена возбуждения и релаксации сигнала фотопроводимости. Такая интерпретация подтверждается результатами измерения кинетики сигнала фотопроводимости, и отсутствием поляризационной зависимости сигнала фотопроводимости. Большое время спада сигнала, равное $\sim 15$ мкс

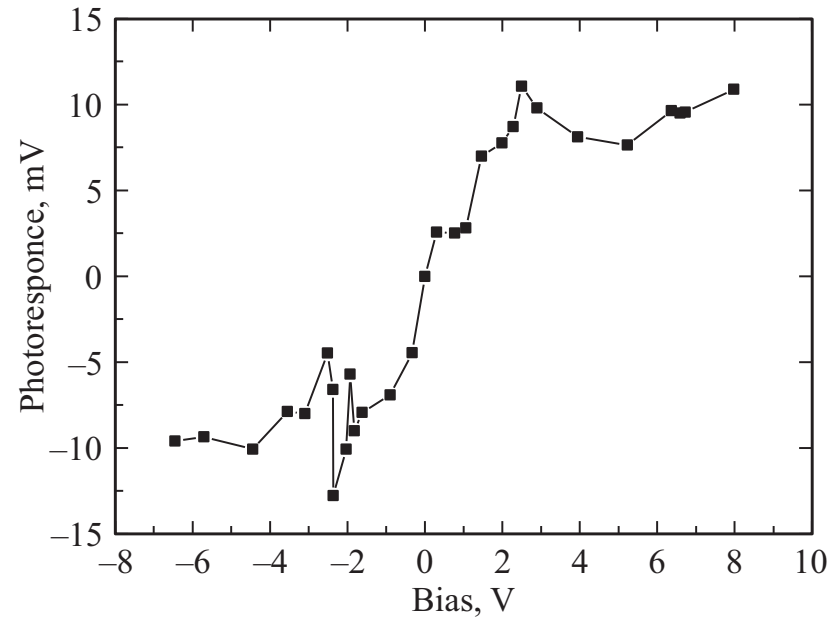

Рис. 2. Зависимость сигнала фотопроводимости, измеренная на эпитаксиальном графене с $p$-n-переходом, как функция приложенного к образцу напряжения.

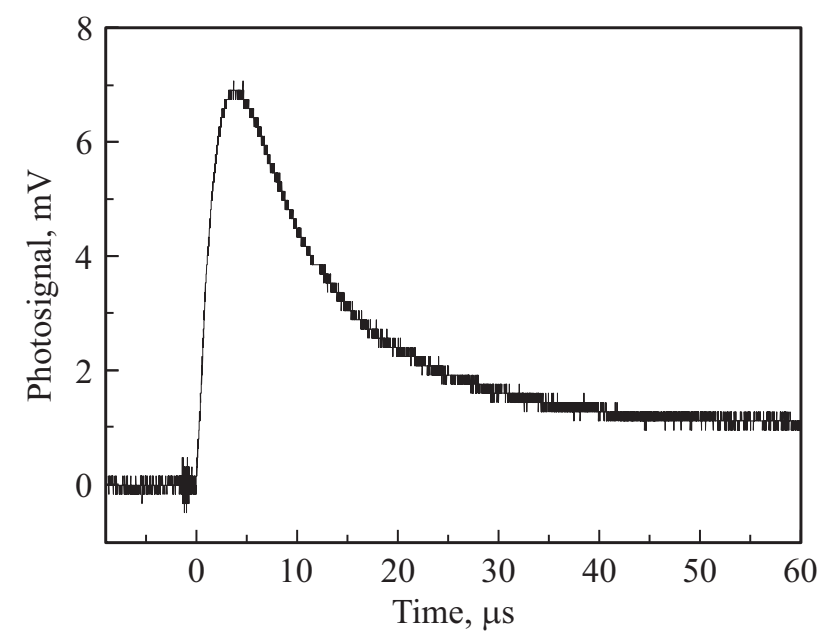

Рис. 3. Кинетика сигнала фотопроводимости, измеренного при смещении 5 В.

(рис. 3), прямо указывает на нагрев образца лазерным излучением. Второй вклад в сигнал может возникать изза облучения части образца под действием лазерного луча, если диаметр пятна лазера меньше, чем размер образца. Если считать, что число носителей заряда не меняется (энергии кванта света не достаточно для создания электронно-дырочных пар), то эффект будет состоять в разогреве части образца. В этом случае появляются неравновесные потоки частиц от горячего пятна в окружающую среду, температура которой ниже. Такой механизм в литературе получил название как фототермоэлектрический эффект [33].

При нулевом напряжении смещения в $p-n$-переходе индуцируется фототок при облучении терагерцовым излучением, как показано на рис. 4 для излучения с длиной волны 280 мкм. Освещая образцы при нормальном падении, мы обнаружили фототок, величина кото- 


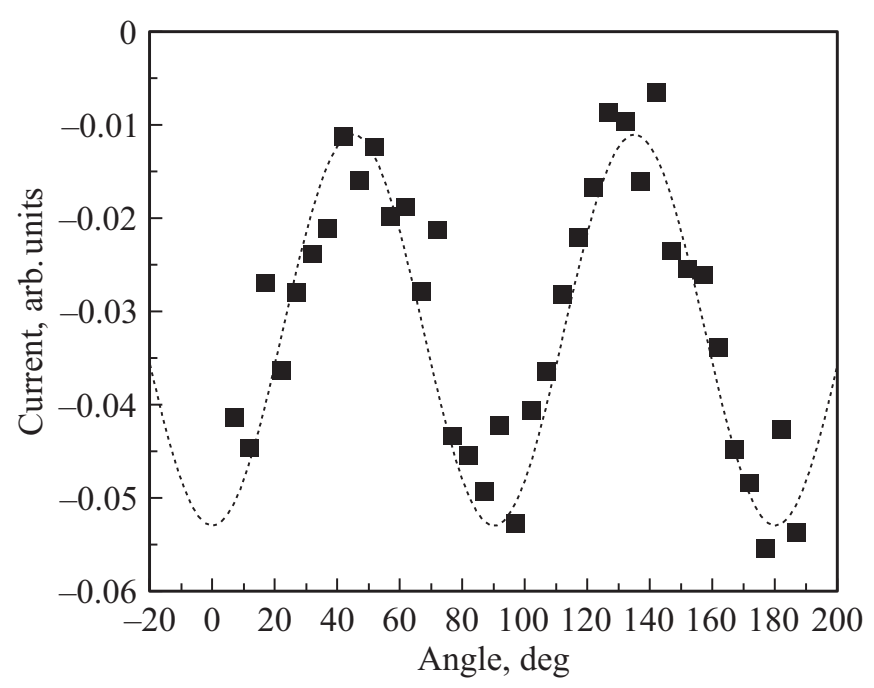

Рис. 4. Фототок, измеренный на эпитаксиальном графене с $p-n$-переходом, в зависимости от угла между направлением $p-n$-перехода и плоскостью поляризации излучения с длиной волны 280 мкм.

рого чувствительна к поляризации излучения. Тот факт, что фотоответ появляется при нормально падающем излучении, может указывать на наличие потенциального барьера. Действительно, в однородном образце сигнал фототока наблюдается только при наклонном падении излучения [34,35]. Дальнейшее экспериментальное проявление $p-n$-перехода было обнаружено в измерениях поляризационной зависимости. Фототок максимален, когда поляризация падающего лазерного излучения составляет 0,180 и $360^{\circ} \mathrm{C}$ (рис. 4), т. е. поляризация перпендикулярна $p-n$-переходу. Это справедливо для обоих образцов независимо от длины волны лазерной генерации. Изменение фототока при вращении угла поляризации, как видно из рис. 4 , велико, $\left(I_{\max }-I_{\min }\right) / I_{\max } \sim 0.7$, что означает, что фототок сильно поляризован. Последнее также удивительно, если сравнивать с предыдущими исследованиями $p-n$-переходов в видимом диапазоне [36], где наблюдались значительно более слабые поляризационные зависимости.

Сильная поляризационная зависимость фототоков не может быть связана с поляризационными свойствами полосковой структуры в наших образцах, так как проводимость образца вдоль и поперек туннельно-прозрачного $p-n$-перехода практически одинаковая. Более того, эксперименты в магнитном поле показали, что направление поляризации фототока сильно меняется с увеличением магнитного поля, что указывает на важность поляризационных свойств самих фототоков, а не структурных особенностей поверхности образца. Таким образом, фототермоэлектрический эффект не объясняет возникновение фототоков, поскольку разогрев носителей практически не зависит от поляризации падающего излучения. В отличие от предыдущих публикаций, где такой эффект индуцировался прямыми межзонными переходами в графене, в наших образцах возможны только непрямые друдевские переходы, что приводит к значительно более слабому поглощению по сравнению с прямыми переходами и соответственно слабому разогреву носителей заряда.

В этом случае можно использовать квазиклассический подход к описанию переноса электронов через $p-n$-переход, и наблюдаемый фотоответ может быть объяснен как результат выпрямления высокочастотного электрического сигнала. Электрическое поле терагерцового излучения, падающего на $p-n$-переход, вызывает высокочастотный электрический ток, который частично выпрямляется из-за нелинейности вольт-амперной характеристики $p-n$-перехода.

\section{4. Заключение}

В работе приведены результаты изготовления и исследования $p-n$-переходов в графене, которые могут использоваться в качестве детекторов терагерцового излучения. Латеральные $p$-n-переходы создавались с помощью засветки ультрафиолетовым излучением монослойного графена, выращенного методом термической сублимации на поверхности $4 H-\mathrm{SiC}(1000)$. Были созданы структуры, длина $p-n$-переходов в которых составляет нескольких сантиметров. Впервые экспериментально измерен терагерцовый отклик $p-n$-переходов в двух режимах: фотопроводимости и фототока. Результаты исследования поляризационной зависимости фотоответа доказывают, что генерация фототока в таких системах происходит за счет выпрямления в $p-n$-переходе, а сигнал фотопроводимости связан с фототермоэлектрическим эффектом при разогреве носителей заряда в результате поглощения терагерцового излучения на непрямых друдевских переходах.

Работа выполнена при частичной поддержке РФФИ 16-02-00854 и DFG (SFB 1277-A04 и GRK1570).

Авторы благодарны С.А. Тарасенко, В.В. Белькову и В.Ю. Качоровскому за обсуждение результатов.

\section{Список литературы}

[1] P.N. First, W.A. de Heer, T. Seyller, C. Berger, J.A. Stroscio, J.-S. Moon. MRS Bulletin, 35 (4), 296 (2010).

[2] A.C. Ferrari, F. Bonaccorso, V. Fal'ko et al. Nanoscale, 7, 4598 (2015).

[3] Yu.B. Vasilyev, G.Yu. Vasileva, Yu.L. Ivanov, S. Novikov, S.N. Danilov. Appl. Phys. Lett., 105 (17), 171105 (2014).

[4] A.A. Dubinov, V.Ya. Aleshkin, M. Ryzhii, T. Otsuji, V. Ryzhii. Appl. Phys. Exp., 2 (9), 092301 (2009).

[5] S. Hertel, D. Waldmann, J. Jobst, A. Albert, M. Albrecht, S. Reshanov, A. Schoner, M. Krieger, H.B. Weber. Nature Commun., 3, 957 (2012).

[6] А.А. Лебедев, С.П. Лебедев, С.Н. Новиков, В.Ю. Давыдов, А.Н. Смирнов, Д.П. Литвин, Ю.Н. Макаров, В.С. Левицкий. ЖТФ, 86 (3), 135 (2016). 
[7] А.А. Лебедев, В.Ю. Давыдов, С.Н. Новиков, Д.П. Литвин, Ю.Н. Макаров, В.Б. Климович, М.П. Самойлович. Письма ЖТФ, 42 (14), 28 (2016).

[8] J.R. Williams, L. DiCarlo, C.M. Marcus. Science, 317 (5838), 638 (2007).

[9] T. Lohmann, K. von Klitzing, J.H. Smet. Nano Lett., 9 (5), 1973 (2009).

[10] B.H. Kim, S.J. Hong, S.J. Baek, H.Y. Jeong, N. Park, M. Lee, S.W. Lee, M. Park, S.W. Chu, H.S. Shin, J. Lim, J.C. Lee, Y. Jun, Y.W. Park. Sci. Rep., 2, 690 (2012).

[11] R. Lv, Q. Li, A.R. Botello-Mendez, T. Hayashi, B. Wang, A. Berkdemir, Q. Hao, A.L. Elias, R. Cruz-Silva, H.R. Gutierrez, Y.A. Kim, H. Muramatsu, J. Zhu, M. Endo, H. Terrones, J.-C. Charlier, M. Pan, M. Terrones. Sci. Rep., 2, 586 (2012).

[12] X. Wang, X. Li, L. Zhang, Y. Yoon, P.K. Weber, H. Wang, J. Guo, H. Dai. Science, 324 (5928), 768 (2009).

[13] Z. Fang, Y. Wang, Z. Liu, A. Schlather, P.M. Ajayan, F.H.L. Koppens, P. Nordlander, N.J. Halas. ACS Nano, 6 (11), 10222 (2012).

[14] D. Wu, K. Yan, Y. Zhou, H. Wang, L. Lin, H. Peng, Z. Liu. J. Am. Chem. Soc., 135 (30), 10926 (2013).

[15] I. Childres, L.A. Jauregui, M. Foxe, J. Tian, R. Jalilian, I. Jovanovic, Y.P. Chen. Appl. Phys. Lett., 97 (17), 173109 (2010).

[16] M. Kalbac, O. Lehtinen, A. Krasheninnikov, J. Keinonen. Adv. Mater., 25 (7), 1004 (2012).

[17] X. Yu, Y. Shen, T. Liu, T.T. Wu, Q.J. Wang. Sci. Rep., 5, 12014 (2015).

[18] T. Mueller, F. Xia, P. Avouris. Nature Photonics, 4, 297 (2010).

[19] E. Vesapuisto, W. Kim, S. Novikov, H. Lipsanen, P. Kuivalainen. Phys. Status Solidi B, 248 (8), 1908 (2011).

[20] D.S. Lee, C. Riedl, B. Krauss, K. von Klitzing, U. Starke, J.H. Smet. Nano Lett. 8, (12), 4320 (2008).

[21] X.S. Wu, Y.K. Hu, M. Ruan, N.K. Madiomanana, J. Hankinson, M. Sprinkle, C. Berger, W.A. de Heer. Appl. Phys. Lett., 95 (22), 223108 (2009).

[22] S. Kopylov, A. Tzalenchuk, S. Kubatkin, V.I. Fal'ko. Appl. Phys. Lett., 97 (11), 112109 (2010).

[23] S. Ryu, L. Liu, S. Berciaud, Y.-J. Yu, H. Liu, P. Kim, G.W. Flynn, L.E. Brus. Nano Lett., 10 (12), 4944 (2010).

[24] S. Lara-Avila, K. Moth-Poulsen, R. Yakimova, T. Bjørnholm, V. Fal'ko, A. Tzalenchuk, S. Kubatkin. Adv. Mater., 23 (7), 878 (2011).

[25] S. Novikov, N. Lebedeva, K. Pierz, A. Satrapinski. IEEE Trans. Instrum. Measurem., 64 (6), 1533 (2015).

[26] S.D. Ganichev, S.N. Danilov, V.V. Bel'kov, E.L. Ivchenko, M. Bichler, W. Wegscheider, D. Weiss, W. Prettl. Phys. Rev. Lett., 88 (5), 057401 (2002.)

[27] V. Lechner, L.E. Golub, P. Olbrich, S. Stachel, D. Schuh, W. Wegscheider, V.V. Bel'kov, S.D. Ganichev. Appl. Phys. Lett., 94 (24), 242109 (2009).

[28] C. Jiang, V.A. Shalygin, V.Yu. Panevin, S.N. Danilov, M.M. Glazov, R. Yakimova, S. Lara-Avila, S. Kubatkin, S.D. Ganichev. Phys. Rev. B, 84 (12), 125429 (2011).

[29] С.Д. Ганичев, С.А. Емельянов, И.Д. Ярошецкий. Письма ЖЭТФ, 35, 297 (1982).

[30] С.Д. Ганичев, Я.В. Терентьев, И.Д. Ярошецкий. Письма в ЖТФ, 11 (1), 46 (1985).

[31] S.D. Ganichev, W. Prettl. Intense Terahertz Excitation of Semiconductors (Oxford University Press, 2006).
[32] V.V. Bel'kov, S.D. Ganichev, P. Schneider, C. Back, M. Oestreich, J. Rudolph, D. Hägele, L.E. Golub, W. Wegscheider, W. Prettl. Sol. St. Commun., 128 (8), 283 (2003).

[33] N.M. Gabor, J.C. Song, Q. Ma, N.L. Nair, T. Taychatanapat, K. Watanabe, T. Taniguchi, L.S. Levitov, P. Jarillo-Herrero. Science, 334 (6056), 648 (2011).

[34] J. Karch, J. Karch, P. Olbrich, M. Schmalzbauer, C. Zoth, C. Brinsteiner, M. Fehrenbacher, U. Wurstbauer, M.M. Glazov, S.A. Tarasenko, E.L. Ivchenko, D. Weiss, J. Eroms, R. Yakimova, S. Lara-Avila, S. Kubatkin, S.D. Ganichev. Phys. Rev. Lett., 105 (22), 227402 (2010).

[35] C. Drexler, S.A. Tarasenko, P. Olbrich, J. Karch, M. Hirmer, F. Müller, M. Gmitra, J. Fabian, R. Yakimova, S. Lara-Avila, S. Kubatkin, M. Wang, R. Vajtai, P.M. Ajayan, J. Kono, S.D. Ganichev. Nature Nanotechnol., 8, 104 (2013).

[36] M. Kim, H.A. Yoon, S. Woo, D. Yoon, S.W. Lee, H. Cheong. Appl. Phys. Lett., 101 (7), 073103 (2012).

Редактор Г.А. Оганесян

\section{Fabrication of graphene p-n junctions and their application for terahertz detection}

\author{
G.Yu. Vasileva ${ }^{1}$, Yu.B. Vasilyev ${ }^{1}$, S.N. Novikov ${ }^{2}$, \\ S.N. Danilov ${ }^{3}$, S.D. Ganichev ${ }^{3}$ \\ ${ }^{1}$ loffe Institute, \\ 194021 St. Petersburg, Russia \\ ${ }^{2}$ Micro and Nanoscience Laboratory, Aalto University, \\ FIN-02150 Espoo, Finland \\ ${ }^{3}$ Institut für Angewandte Physik, \\ Universität Regensburg, \\ D-38106 Regensburg, Germany
}

Abstract A new method for the lateral $p-n$-junctions formation in epitaxial graphene by UV radiation is considered. The UV illumination method allows to obtain large-scale $p-n$-junctions, which were investigated in the photocurrent and photoconductivity regimes upon irradiation by terahertz radiation. The mechanisms of the terahertz photoresponse in graphene $p-n$-junctions are discussed. 